\title{
OPTIMAL FILTER SYSTEMS FOR PHOTOMETRIC REDSHIFT ESTIMATION
}

\author{
N. Benítez ${ }^{1,2}$, M. Moles ${ }^{1}$, J. A. L. Aguerri ${ }^{3}$, E. Alfaro ${ }^{1}$, T. Broadhurst ${ }^{4}$, J. Cabrera-Caño ${ }^{5}$, F. J. Castander ${ }^{6}$, J. Cepa ${ }^{3}$, \\ M. Cerviñõo ${ }^{1}$, D. Cristóbal-Hornillos ${ }^{1}$, A. Fernández-Soto ${ }^{7,8}$, R. M. González Delgado ${ }^{1}$, L. Infante ${ }^{9}$, I. Márquez ${ }^{1}$, \\ V. J. Martínez ${ }^{7}$, J. Masegosa ${ }^{1}$, A. Del Olmo ${ }^{1}$, J. Perea ${ }^{1}$, F. Prada ${ }^{1}$, J. M. Quintana ${ }^{1}$, and S. F. Sánchez ${ }^{10}$

\begin{abstract}
In the coming years, several cosmological surveys will rely on imaging data to estimate the redshift of galaxies, using traditional filter systems with 4-5 optical broad bands; narrower filters improve the spectral resolution, but strongly reduce the total system throughput. We explore how photometric redshift performance depends on the number of filters $n_{f}$, characterizing the survey depth by the fraction of galaxies with unambiguous redshift estimates. For a combination of total exposure time and telescope imaging area of $270 \mathrm{hr} \mathrm{m}^{2}, 4-5$ filter systems perform significantly worse, both in completeness depth and precision, than systems with $n_{f} \gtrsim 8$ filters. Our results suggest that for low $n_{f}$ the color-redshift degeneracies overwhelm the improvements in photometric depth, and that even at higher $n_{f}$ the effective photometric redshift depth decreases much more slowly with filter width than naively expected from the reduction in the signal-to-noise ratio. Adding near-IR observations improves the performance of low- $n_{f}$ systems, but still the system which maximizes the photometric redshift completeness is formed by nine filters with logarithmically increasing bandwidth (constant resolution) and half-band overlap, reaching $\sim 0.7$ mag deeper, with $10 \%$ better redshift precision, than 4-5 filter systems. A system with 20 constant-width, nonoverlapping filters reaches only $\sim 0.1$ mag shallower than 4-5 filter systems, but has a precision almost three times better, $\delta z=0.014(1+z)$ versus $\delta z=$ $0.042(1+z)$. We briefly discuss a practical implementation of such a photometric system: the ALHAMBRA Survey.
\end{abstract}

Key words: cosmology: observations - galaxies: distances and redshifts - instrumentation: miscellaneous surveys

\section{INTRODUCTION}

Photometric redshift (photo- $z$ ) estimation is not a new technique (Baum 1962; Loh \& Spillar 1986; Koo 1985; Connolly et al. 1995; see Koo 1999 for a history of the method), but it has considerably developed in the last decade, especially following the Hubble Deep Field (HDF) observations (Williams 1996; Casertano et al. 2000), which provided catalogs with excellent photometric quality and abundant spectroscopic redshift coverage. This allowed astronomers to thoroughly test standard photo- $z$ techniques and try new approaches (Gwyn \& Hartwick 1996; Lanzetta et al. 1996; Sawicki et al. 1997; Fernández-Soto et al. 1999; Brunner et al. 1997; Benítez et al. 1999; Benítez 2000; Bolzonella et al. 2000).

As Hickson et al. (1994) first showed, multiband narrow filters can be much more efficient than spectroscopy for obtaining redshifts if the large area of the imaging cameras is factored in. Several photometric surveys, using different filter systems, have been proposed or implemented in the last decade: the UBC-NASA survey (Hickson \& Mulrooney 1998), CADIS (Wolf et al. 2001b), COMBO-17 (Wolf et al. 2001a), COSMOS-21 (Taniguchi 2004), Advanced, Large, Homogeneous Area, Medium Band Redshift Astronomical (ALHAMBRA; Moles et al. 2008), DES (DES Collaboration 2005), LSST (Tyson 2006), PanStarrs (Kaiser 2007), VST (Arnaboldi et al. 2007), and PAU (Benítez et al. 2009). These surveys repre- sent powerful alternatives to deep spectroscopic surveys such as DEEP2 (Davis et al. 2003), VVDS (Le Fèvre et al. 2003), or BOSS (Schlegel et al. 2007) at least for those scientific goals which only require limited redshift accuracy and low-resolution spectral information.

However, at least three of the imaging surveys (DES, LSST, PanStarrs) will work with photometric systems with 4-5 optical broadband filters, similar to those traditionally used in astronomy. It is obvious that using more, narrower, filters for a fixed exposure time will significantly sacrifice photometric depth. However, photometric depth is not equivalent to photometric redshift depth. The fewer the filters, the more prone the system is to color-redshift degeneracies; these make it impossible to unambiguously determine the redshift for a galaxy, even if observed at relatively high signal-to-noise ratio $(\mathrm{S} / \mathrm{N})$. The Hubble Ultra Deep Field (HUDF; Beckwith et al. 2003) offers a good example. Despite the fact that the limiting magnitude in the HUDF is 0.9-1.4 mag deeper than the HDF, the lack of a $U$-band filter in the HUDF makes the photometric redshift depth of both fields similar (Coe et al. 2006).

This Letter explores the impact on photometric redshift performance of factors such as the number of filters $n_{f}$, constant versus logarithmically increasing bandwidth, half-band overlaps, and near-IR observations. We also briefly discuss a practical implementation of a medium band filter system: the ALHAMBRA Survey. 


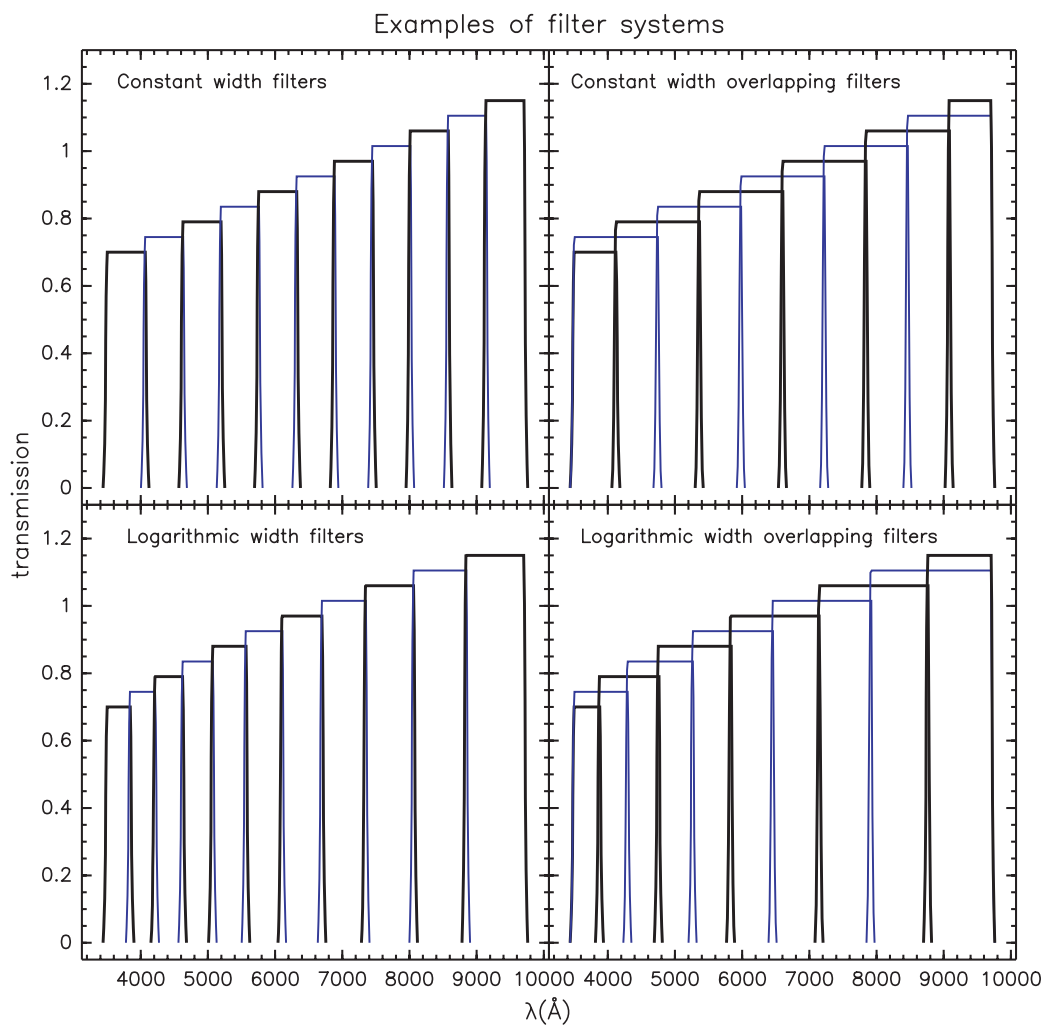

Figure 1. Example of the four types of filter sets considered, each with 11 filters. We represent the filter transmissions without taking into account the CCD or the telescope + optics transmission, factors which are later included in the photometric noise estimation to produce realistic photometric measurements. We slightly increase the height of the filters with wavelength, and alternate colors in successive filters to help visualization.

\section{SIMULATIONS}

\subsection{Description of the Filter Systems}

We assume that filters are almost "top hat," with a transmission which is constant in the central part and steeply falls on the edges, formed by half-Gaussian wings with a half-width at half-maximum of $\sim 15 \AA$. Although somewhat idealized, this is very similar to the characteristics of the filters provided by BARR Associates for the ALHAMBRA Survey. We consider four types of photometric systems, depending on whether they have constant or logarithmically increasing $(\Delta \lambda \sim \lambda)$ bandwidth, and whether they have half-width $\Delta \lambda / 2$ overlaps or just a minimal overlap corresponding to the filter wings. The filters cover the 3400-9800 $\AA$ interval. Figure 1 shows examples of the four types of filter systems considered.

\subsection{Mock Catalogs}

To generate realistic galaxy distributions, we use the HDF catalogs of Fernández-Soto et al. (1999) and Yahata et al. (2000). It is possible to obtain accurate and reliable photometric redshifts and type classifications, based on the extended Coleman et al. (1980) set for these galaxies up to $I_{\mathrm{AB}} \approx 27$ (Benítez 2000; Fernández-Soto et al. 2001). We use the distribution of $I_{\mathrm{AB}}$, Bayesian spectral type $t_{b}$, and Bayesian photometric redshift $z_{b}$ obtained from these catalogs with the software BPZ (http://acs.pha.jhu.edu/ txitxo; Benítez 2000) and the empirically calibrated spectral library of Benítez et al. (2004). The original input catalogs contain 822 galaxies with $I_{\mathrm{AB}}<26$. For each filter set combination, our simulation includes 5000 galaxies, generated by randomly choosing objects from the HDF catalogs. The depth reached by our simulations (up to $I \sim 25$ ) precludes using an input catalog based on spectroscopic redshifts, which would not be complete enough at those magnitudes. Since the accuracy of the input photometric redshifts is $\approx 0.06(1+z)$, we perturb them by a similar, randomly distributed amount to produce a more realistic redshift distribution.

We assume a constant total exposure time of $100,000 \mathrm{~s}$ $\left(28 \mathrm{hr}\right.$ ) per pointing, and vary the number of filters $n_{f}$. The average exposure is thus $100,000 / n_{f}$. Instead of using a fixed observing time per filter, we distribute the exposures trying to reach constant $\mathrm{S} / \mathrm{N}$ for the same $\mathrm{AB}$ magnitude, with two constraints: the minimal exposure time per filter is, for practical reasons, at least $2500 \mathrm{~s}$, and we do not expose more than twice the length of the average exposure, i.e. $2 \times 100,000 / n_{f}$, to avoid spending too much of the total time on the filters which are less efficient. For wavelengths $\lambda>8000 \AA$, the efficiency is so low that we set this limit to be $100,000 / n_{f}$.

Using this information, we generate the expected magnitudes in all the filter systems described in the above section using functions included in the BPZ package. For $\sim 300 \AA$ filters, the corresponding $5 \sigma$ limiting magnitudes are $m_{\mathrm{AB}} \sim 25$ blueward of $8000 \AA$ and quickly degrades to $m_{\mathrm{AB}} \sim 23$ at $9500 \AA$. As is obvious, for a fixed total exposure time, the limiting flux in a filter will be roughly inversely proportional to the square root of the filter width $\Delta \lambda$.

As a last step, we add random photometric noise whose amplitude has been estimated using the WHT exposure time calculator ${ }^{11}$ scaling it to a $3.5 \mathrm{~m}$ telescope. The product of exposure time by telescope area is $\sim 270 \mathrm{hr} \mathrm{m}^{2}$, and therefore the results obtained here are equivalent to, e.g., what could be obtained in a few hours of total exposure time with an $8-10 \mathrm{~m}$ telescope.

\footnotetext{
11 Based on the SIGNAL software, by Chris Benn.
} 


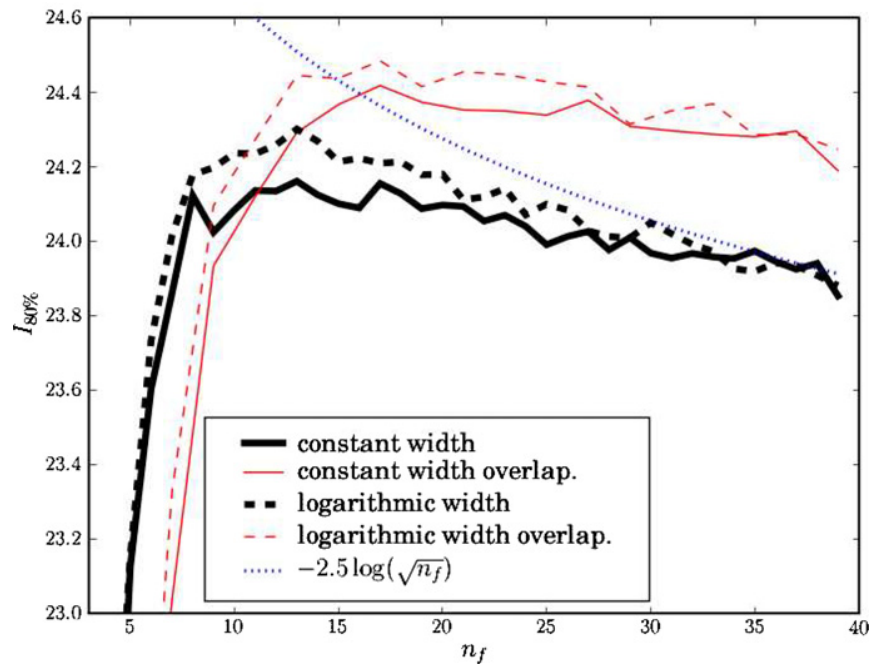

Figure 2. Effective $80 \%$ completeness magnitude, corresponding to the magnitude at which the accumulated number of objects $N\left(<m_{0.99}\right)$ with Bayesian odds $\geqslant 0.99$ is $80 \%$ of the total number of objects $N(<m)$, a good measure of the effective depth of a survey. The blue dotted line illustrates how the completeness magnitude would change with filter number if it mimicked the behavior of the photometric limiting magnitude.

We also scale the $\mathrm{S} / \mathrm{N}$ as a function of the magnitude of the galaxies. We do not take into account the dependence of size, etc., with magnitude. These are second-order effects which will have a similar impact on all the filter systems and, therefore, are not expected to significantly affect the comparison among them.

\section{COMPARISON AMONG DIFFERENT FILTER SYSTEMS}

The photometric redshifts for the mock catalogs are estimated using the BPZ package. The software provides a Bayesian estimate of the redshift and a spectral type classification. The expected reliability of the photo- $z$ can be gauged through the Bayesian odds. The value of this parameter corresponds to the amount of redshift probability concentrated on a $\pm 0.2(1+z)$ region around the probability maximum. Low values of the odds indicate a multimodal or very extended, little informative $p(z)$, indicating that the photometric information is insufficient to obtain an unambiguous estimate of the galaxy redshift. By selecting objects with high odds, e.g. $\geqslant 0.99$, one can produce highly reliable samples (Benítez 2000), with very good redshift accuracy and a very low rate $(\lesssim 2 \%)$ of "catastrophic" outliers. Therefore, it is possible to accurately characterize the effective completeness of a photometric redshift catalog by using the amount of galaxies with odds above a certain threshold, which tells us how many galaxies we can expect to have meaningful, univocal photometric redshifts.

For a setup with a total exposure time $T$ and total number of filters $n_{f}$, the $\mathrm{S} / \mathrm{N}$ in an individual filter, assuming that we are limited by the sky background, would roughly change as $\mathrm{S} / \mathrm{N}_{i} \propto$ $1 / n_{f}$. A way of comparing depths across different systems is the $\mathrm{S} / \mathrm{N}_{B}$ in a fixed-width band (obtained by combining all the individual filters included in that band): $\mathrm{S} / \mathrm{N}_{B} \propto$ $1 / \sqrt{n_{f}}$. Therefore, by increasing the number of filters we would expect the effective limiting magnitude at a fixed $\mathrm{S} / \mathrm{N}$ level to diminish quite drastically, as $m_{\lim }=$ const. $+2.5 \log \left(\sqrt{n_{f}}\right)$, e.g., equivalent to the loss of a full magnitude going from 4 to 25 filters.

Figure 2 describes how the $80 \%$ completeness magnitude limit behaves for each of the filter systems. We see that for

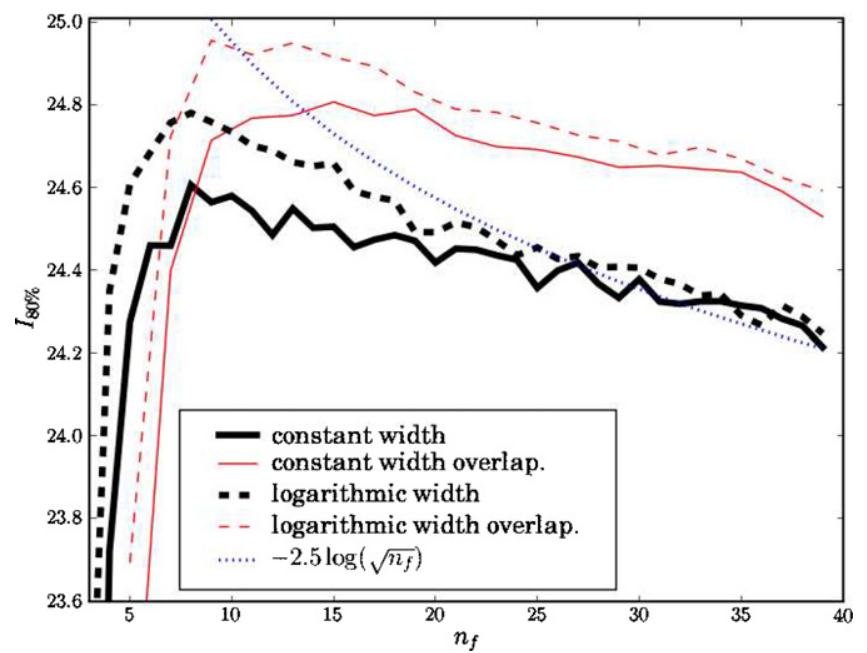

Figure 3. Same as Figure 2, but including moderately deep near-IR observations (see the text for details).

contiguous filters the completeness depth sinks fast for $n_{f}<8$, and that the optimum number of filters is $n_{f} \sim 12$ after which the effective completeness magnitude decreases, but much more slowly than expected from the change in the photometric limiting magnitude. This shows that for systems with low $n_{f}$ the color-redshift degeneracies introduced by an insufficient wavelength resolution dominate over the improvement in the $\mathrm{S} / \mathrm{N}$ achieved by the increased filter width.

Figure 3 shows what happens when we add moderately deep near-IR observations with $5 \sigma$ limiting (Vega) magnitudes of $J=22.4, H=21.2, K=20.4$. There is a very significant, almost $\sim 0.4$ mag, increase in the completeness magnitude, and the behavior of the low- $n_{f}$ systems relatively improves, but still the most efficient overall performer is a logarithmically increasing bandwidth, half-band overlapping system with nine filters, which reaches a completeness limit $\sim 0.7$ mag deeper than a typical 4-5 filter system with the same exposure time, while having a $10 \%$ better accuracy.

Another obvious quantity to consider is the accuracy of the photometric redshifts for the high "odds" sample, estimated using the "rms" of the quantity $\Delta z /(1+z)=\left(z-z_{b}\right) /(1+z)$, plotted in Figure 4. Here we see that, as expected, the redshift precision quickly and monotonously improves with $n_{f}$ and that adjacent filter systems perform much better than overlapping ones. From Figure 3, we can see that an adjacent system with $n_{f}=20$ reaches a completeness depth similar to traditional systems with $n_{f}=5$, but an accuracy significantly better: $0.015(1+z)$ versus $0.04(1+z)$.

\section{THE ALHAMBRA SURVEY}

Considering the above results, plus additional requirements on emission line detection, the ALHAMBRA Survey decided to use a constant-width, nonoverlapping filter system, complemented with near-IR observations. ALHAMBRA had an additional requirement, the detection of a large fraction of galaxies with emission lines, which favored the choice of $310 \AA$ filters. The ALHAMBRA $3 \sigma$ rest-frame detection limits for a typical $\mathrm{AB} \approx 23$ galaxy are $\mathrm{EW}(\mathrm{H} \alpha)>28 \AA$ out to $z \approx 0.45$, and $\mathrm{EW}(\mathrm{O}$ II $)>16 \AA$ out to $z \approx 1.55$. From comparison with Hippelein et al. (2003), ALHAMBRA expects to detect $\approx 50 \%$ of the $\mathrm{H} \alpha$ emitters at $z \approx 0.25$, and $\approx 80 \%$ of the $\mathrm{O}$ II objects at $z \approx 1.2$; since $80 \%$ of the field galaxies at those redshifts have 


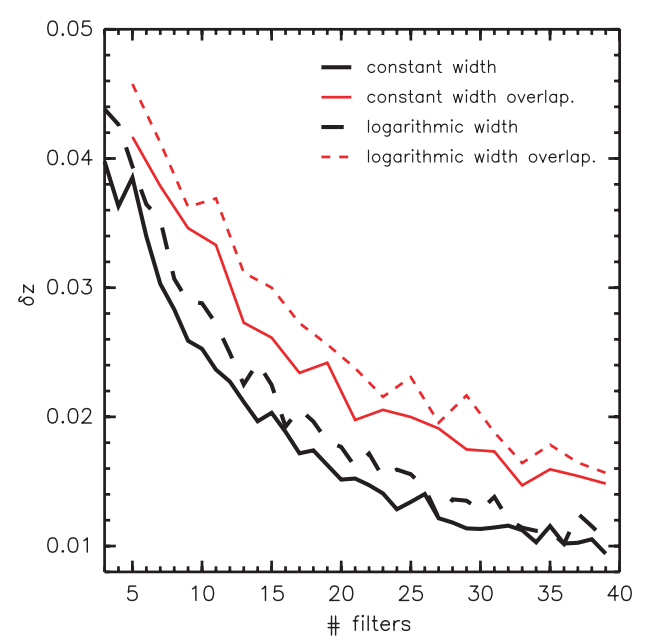

Figure 4. Dependence of the rms of quantity $\left(z-z_{b}\right) /(1+z)$ for those galaxies with odds $>0.99$ as a function of the number of filters for the four types of filter systems considered in the Letter and including near-IR observations (see the text for details).

detectable emission lines (Tresse \& Maddox 1998), we expect to detect lines for a large fraction of our whole sample.

The survey is imaging $4 \mathrm{deg}^{2}$ with the camera LAICA at the Calar Alto $3.5 \mathrm{~m}$ telescope and also obtaining deep $J H K$ observations with Omega2000 at the same telescope. The survey characteristics, scientific goals, and preliminary results are described in detail in Moles et al. (2008). A good test of the simulations presented in this Letter is a comparison with ALHAMBRA. The mock catalogs predict that, with 20 filters, ALHAMBRA should be able to reach a precision of $\delta z /(1+z) \approx 0.014$ for $I \lesssim 24$ galaxies. Preliminary results show that the measured redshift error (Moles et al. 2008) is similar or less than 0.015 , supporting the validity of the simulations presented in this Letter.

\section{CONCLUSIONS}

We explore the performance of four different uniform filter systems with constant and logarithmically increasing $(\Delta \lambda \propto \lambda)$ widths, and with half-width $\Delta \lambda / 2$ overlaps or just a minimal overlap corresponding to the filter wings, and use, as a measure of survey-effective depth, the fraction of galaxies with a compact, unimodal probability redshift distributions as a function of magnitude. Our simulations employ a realistic input catalog, based on HDF photometric redshifts, and correspond to a combination of total exposure time and telescope area of $270 \mathrm{hr} \mathrm{m}^{2}$. We find that traditional 4-5 optical filter systems clearly underperform, both in terms of completeness magnitude limit and precision, systems with $n_{f} \gtrsim 8$ filters.

Our results suggest that for low $n_{f}$ the effect of color-redshift degeneracies dominates the advantages of increased photometric depth, and that even at higher $n_{f}$ the effective photometric redshift depth decreases much more slowly with filter width than naively expected from the reduction in the $\mathrm{S} / \mathrm{N}$. Adding nearIR observations increases the overall depth, alleviating colorredshift degeneracies, and improving the relative performance of low- $n_{f}$ systems. However, the optimum performance still comes from a system with nine filters with logarithmically increasing bandwidth (constant resolution) and half-band overlap, which reaches $\sim 0.7$ mag deeper, with $10 \%$ better redshift precision than 4-5 filter systems. For many scientific applications, which require both precision and depth, the use of $>15$ medium band filters is clearly advantageous. A system with 20 constant- width, nonoverlapping filters reaches only $\sim 0.1$ mag shallower than 4-5 filter systems, but has a precision almost 3 times better, $\delta z=0.014(1+z)$ versus $\delta z=0.042(1+z)$, as a practical implementation of such a system, the ALHAMBRA Survey, shows.

Since it is well known that color-redshift degeneracies worsen with magnitude depth, it can be expected that the relative decoupling between photometric depth and photometric redshift depth described here will be more significant for surveys which reach fainter limits than those considered in our simulations, and less important for shallower observations, where the color/ redshift degeneracies are less of a problem. In any case, future projects will have to seek an optimum number of filters based on their particular observing parameters and science goals.

This work has been supported by the CONSOLIDER AYA2006-14056 and the proyecto intramural del CSIC $200750 \mathrm{I003}$.

\section{REFERENCES}

Arnaboldi, M., Neeser, M. J., Parker, L. C., Rosati, P., Lombardi, M., Dietrich, J. P., \& Hummel, W. 2007, The Messenger, 127, 28

Baum, W. A. 1962, in IAU Symp. 15, Problems of Extra-Galactic Research, ed. G. C. McVittie (New York: Macmillan), 390

Beckwith, S. V. W., et al. 2003, BAAS, 35, 723

Benítez, N. 2000, ApJ, 536, 571

Benítez, N., Broadhurst, T., Bouwens, R., Silk, J., \& Rosati, P. 1999, ApJ, 515 , L65

Benítez, N., et al. 2004, ApJS, 150, 1

Benítez, N., et al. 2009, ApJ, 691, 241

Bolzonella, M., Miralles, J.-M., \& Pelló, R. 2000, A\&A, 363, 476

Brunner, R. J., Connolly, A. J., Szalay, A. S., \& Bershady, M. A. 1997, ApJ, 482, L21

Casertano, S., et al. 2000, AJ, 120, 2747

Coe, D., Benítez, N., Sánchez, S. F., Jee, M., Bouwens, R., \& Ford, H. 2006, AJ, 132,926

Coleman, G. D., Wu, C.-C., \& Weedman, D. W. 1980, ApJS, 43, 393

Connolly, A. J., Csabai, I., Szalay, A. S., Koo, D. C., Kron, R. G., \& Munn, J. A. 1995, AJ, 110, 2655

Davis, M., et al. 2003, Proc. SPIE, 4834, 161

Fernández-Soto, A., Lanzetta, K. M., Chen, H., Pascarelle, S. M., \& Yahata, N. 2001, ApJS, 135, 41

Fernández-Soto, A., Lanzetta, K. M., \& Yahil, A. 1999, ApJ, 513, 34

Gwyn, S. D. J., \& Hartwick, F. D. A. 1996, ApJ, 468, L77

Hickson, P., Gibson, B. K., \& Callaghan, K. A. S. 1994, MNRAS, 267, 911

Hickson, P., \& Mulrooney, M. K. 1998, ApJS, 115, 35

Hippelein, H., et al. 2003, A\&A, 402, 65

Kaiser, N. 2007, Am. Astron. Soc. Meeting Abstr., 210, 51.02

Koo, D. C. 1985 , AJ, 90, 418

Koo, D. C. 1999, in ASP Conf. Ser. 191, Photometric Redshifts and the Detection of High Redshift Galaxies, ed. R. Weymann, L. Storrie-Lombardi, M. Sawicki, \& R. Brunner (San Francisco, CA: ASP), 3

Kuehn, K., \& Dark Energy Survey Collaboration 2009, Am. Astron. Soc. Meeting Abstr., 213, 301.06

Lanzetta, K. M., Yahil, A., \& Fernández-Soto, A. 1996, Nature, 381, 759

Le Fèvre, O., et al. 2003, The Messenger, 111, 18

Loh, E. D., \& Spillar, E. J. 1986, ApJ, 303, 154

Moles, M., et al. 2008, AJ, 136, 1325

Sawicki, M. J., Lin, H., \& Yee, H. K. C. 1997, AJ, 113, 1

Schlegel, D. J., et al. 2007, Am. Astron. Soc. Meeting Abstr. 211, 132.29

Taniguchi, Y. 2004, in Proc. Japan-German Seminar, Studies of Galaxies in the Young Universe with New Generation Telescope, ed. N. Arimoto \& W. Duschl, 107

Tresse, L., \& Maddox, S. J. 1998, ApJ, 495, 691

Tyson, J. A. 2006, in AIP Conf. Ser. 870, Intersections of Particle and Nuclear Physics, 9th Conf. CIPAN, 4452

Williams, R. E., et al. 1996, AJ, 112, 1335

Wolf, C., Meisenheimer, K., \& Roser, H.-J. 2001a, A\&A, 365, 660

Wolf, C., et al. 2001b, A\&A, 365, 681

Yahata, N., Lanzetta, K. M., Chen, H.-W., Fernández-Soto, A., Pascarelle, S. M., Yahil, A., \& Puetter, R. C. 2000, ApJ, 538, 493 